\title{
2.2 Anwendungsorientierte Regionalforschung - eine Chance für die Geographie?
}

\subsubsection{Problemstellung}

Theorie und tatsächliche Geschichte zeigen in aller Deutlichkeit, daß «vom lokalen bis zum internationalen Maßstab räumliche Differenzierungs- und Polarisierungsprozesse grundsätzlich nicht vermeidbar waren und sind, wenn und solange man überhaupt Entwicklung der menschlichen Gesellschaft befürwortet) (BARTELS 1977). Auch in Zukunft werden als unerwünscht empfundene räumliche Disparitäten und erwünschte Differenzierungen die Diskussion in Wissenschaft, Politik und Praxis beleben. Die Vehemenz dieser Auseinandersetzung wird (wie bisher) phasenweise zu- und wieder abnehmen und den damit verbundenen Wertewandel der verschiedenen beteiligten Akteure widerspiegeln.

Regionalforschung versucht nun, Strukturen, Funktionen und Prozesse regionaler Entwicklungen zu beschreiben, zu erklären, zu bewerten und in ihrer Beeinflußung bzw. Beeinflußbarkeit durch Politik und unterschiedliche Akteure zu untersuchen. Je anwendungsorientierter sie betrieben wird - und die folgenden Ausführungen gründen auf einer explizit anwendungsorientierten Interpretation von geographischer Forschung -, desto eher versucht sie, die genannten unterschiedlichen Phasen im wissenschaftlichen Erkenntnisprozeß (und auch in der Verantwortlichkeit von Wissenschaft) $\mathrm{zu}$ verbinden: anwendungsorientierte Regionalforschung nimmt in diesem Sinne Stellung zu Entwicklungsproblemen in und zwischen Regionen. Ihr Interesse gilt insbesondere der Antizipation von Problemen der Unter-, Fehl- und Überentwicklung.

Diese ausgeprägte Problemorientierung hat natürlich ihre Konsequenz: einschlägige Studien müssen ihre Ziel- und Werte-Orientierung explizit darstellen. Der Grund hierfür ist einfach: Probleme definieren sich v. a. aus Zielen und Werten; diese Wechselbeziehung muß demnach klar sein, falls das Prinzip der Nachvollziehbarkeit für wissenschaftliche Arbeiten nicht verletzt werden soll (STIENS 1980; EISEL 1981).

\subsubsection{Zentrale Forschungsfelder}

Unabhängig davon, ob Regionalforschung in Ländern der Ersten, Zweiten, Dritten oder Vierten Welt angelegt wird, kann von folgendem Grundschema regionaler Entwicklung ausgegangen werden (BRUGGER 1981/1982):
Abb.1 Regionale Entwicklung: ein Grundmodell

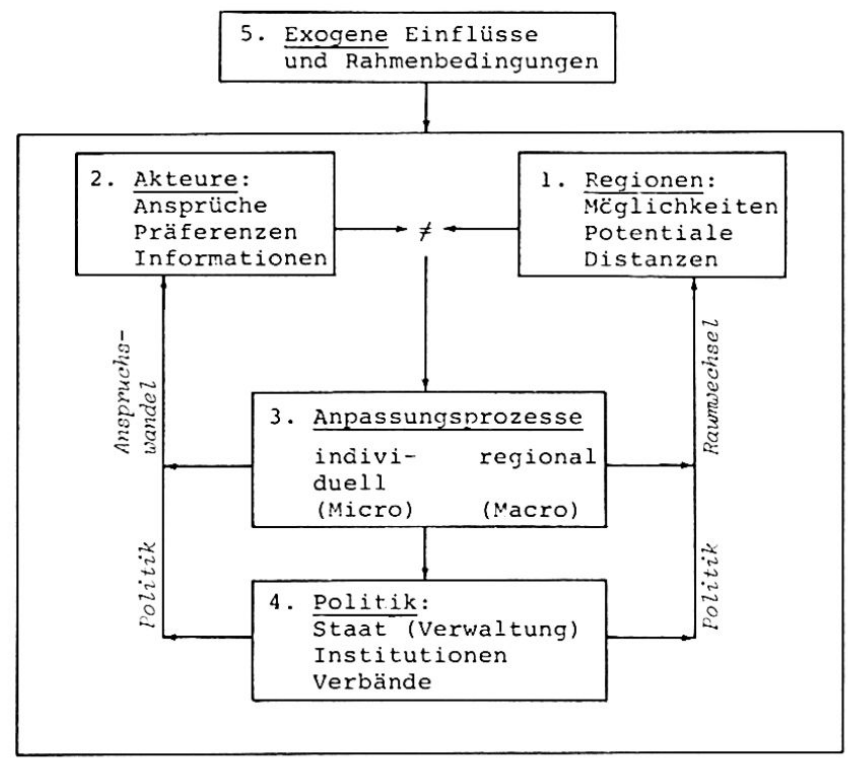

Quelle: Weiterentwicklung aus BRUGGER 1981

Die Regionen besitzen unterschiedlich günstige $M \ddot{g} g$ lichkeiten und Potentiale für das Handeln und Verhalten des Menschen (Feld 1). Die (im Zeitablauf sich ändernde) Beurteilung solcher Möglichkeiten basiert auf Ansprüchen, Präferenzen, Zwängen und Informationen der individuellen und kollektiven Akteure (Feld 2). Falls bei gegebenem Informationsstand eine Diskrepanz zwischen Feld 1 und Feld 2 be- oder entsteht, kommt es in der Regel zu Anpassungsprozessen (Feld 3): diese sind einerseits individueller Art, indem beispielsweise Haushaltungen ihren Wohnort oder Unternehmungen ihren Standort wechseln (Pfeil «Raumwechsel»), oder indem individuelle Akteure ihre Werte und Präferenzen an die bestehenden Möglichkeiten anpassen (Pfeil: "Anspruchswandel»). Anderseits bewirkt die Summe solcher individueller Entscheidungen auch Anpassungsprozesse auf regionaler und interregionaler und damit auf kollektiver Ebene: Wanderungsbilanzen und regionale Identitäts-

Ernst A. Brugger, PD Dr., Programmleiter NFP

"Regionalprobleme"

Bundesgasse 8, $\mathrm{CH}-3003$ Bern 
muster verändern sich. Auf solche Anpassungsprozesse nimmt nun die Politik (Feld 4 und Pfeile «Politik») bewußt und unbewußt Einfluß: mittels Infrastrukturinvestitionen beeinflußt sie beispielsweise Möglichkeiten und Potentialwerte von Regionen, in zahlreichen Programmen fördert und hindert sie bestimmte Werte, Ziele, Präferenzen. Auf alle bisher genannten Felder und ihre Beziehungen wirken exogene Bedingungen und Einflüsse ein: keine Region und kein Land dieser Welt kann als Insel gelten. Zu stark sind beispielsweise weltweite Technologieschübe und großräumige Ökologieprobleme mit regionaler Entwicklung verknüpft, als daß das Zusammenwirken von endogenen und exogenen Prozessen nicht von größtem Interesse sein muß.

Die fünf Felder sind einzelweise und in ihrem Zusammenhang für das Verständnis regionaler Entwicklungsprobleme zentral. Ein Beispiel mag dies verdeutlichen: Die schweizerischen Regionen besitzen unterschiedlich günstige Voraussetzungen (vgl. Feld 1) für technologische Anpassungsprozesse in der Industrie (BRUGGER 1984), während gleichzeitig die Ansprüche und die Informationskapazitäten der Betriebe (vgl. Feld 2) nach bestimmten Merkmalen stark variieren (NYDEGGER u.a. 1983). Anpassungsprozesse (vgl. Feld 3) in Betrieben und Unternehmungen einerseits sowie in Regionen anderseits sind die Folge: tiefgreifende Umwälzungen auf regionalen Arbeitsmärkten sind in der Schweiz insbesondere in der Jurakette zu verzeichnen (MaILlat 1983). Die Politik (vgl. Feld 4) versucht mittels verschiedener Maßnahmen, auf solche Anpassungsprozesse einzuwirken (BRUGGER 1984). Die Wirkung solcher Maßnahmen wird jedoch durch exogene Rahmenbedingungen (Feld 5) stark beeinflußt, so wie auch der Anpassungsdruck selbst zum guten Teil weltweiter Natur ist (STUCKEY 1980).

Dieses Beispiel illustriert auch eine wichtige Schlußfolgerung aus dieser Diskussion: jedes der fünf Felder kann als eigenes Forschungsfeld bezeichnet werden. Regionalforschung beinhaltet demnach beispielsweise Fragestellungen in den Bereichen

- Potentialanalyse (Feld 1)

- Präferenzstrukturen (Feld 2)

- Standortverlagerungen und Mobilität (Feld 3)

- Wirkungsanalysen (Feld 4)

- Internationale Arbeitsteilung (Feld 5).

Zwei weitere Folgerungen lassen sich daraus ziehen: einerseits wird klar, daß nicht nur die einzelnen Felder von Interesse sind, sondern insbesondere auch die Zusammenhänge zwischen diesen Feldern; und anderseits ergibt sich eine klare Herausforderung an die Wissenschaft, von der Problemstellung und nicht von scharf beobachteten Disziplingrenzen her Forschung zu betreiben: die Probleme regionaler Entwicklung und Entwicklungspolitik sind nur mittels Interdisziplinarität und «vernetztem Denken» (RAPOPORT 1982) lösbar.

\subsubsection{Chance für die Geographie?}

Kann nun so verstandene anwendungsorientierte Regionalforschung als Chance für die Geographie bezeichnet werden? Ohne Zweifel beinhaltet jedes der fünf Felder Fragestellungen, die aus geographischer Sicht anzugehen wären. $\mathrm{Zu}$ den Feldern 1 und 2 liegen recht viele einschlägige Arbeiten vor, wesentlich weniger $\mathrm{zu} 3$, fast keine zu 4 und 5 . Zusammenhänge zwischen den Feldern werden durch Geographen wenig diskutiert. Ein Potential an ungelösten Fragen liegt also vor, die zum Teil durch Geographen behandelt werden könnten. Zumindest die deutschsprachige Geographie hat hier zahlreiche Gelegenheiten verpaßt und ist folgerichtig praktisch aus dem «Markt» um Forschungsgelder und um Aufmerksamkeit von Medien und Politik verdrängt worden.

Die Hoffnung auf eine Trendwende sollte allerdings nicht zu früh aufgegeben werden. Sie ist jedoch an zumindest vier Voraussetzungen gebunden:

- Das Fach müßte seine Kontaktintensität zu Praxis und Politik merklich steigern, nicht zuletzt um wesentliche Fragestellungen frühzeitig erkennen zu können.

- Das Fach müßte eine Art regionalwissenschaftliches Forschungsprogramm entwickeln, das mit seiner mittelfristigen Orientierung als Koordinationshilfe innerhalb von und zwischen Instituten und Gruppen dienlich wäre.

- Theoretisch und metatheoretisch müßte der Hintergrund des Faches deutlich verstärkt werden. Gerade eine anwendungsorientierte Forschungsrichtung ist auf klare theoretische Konzepte angewiesen und muß ihre normativen Schlüsselstellen explizieren.

- Bei aller notwendigen intradisziplinären Spezialisierung müßte die Geographie neuen Mut zur Synthese entwickeln. Die Problemstellungen sind komplex und vernetzt. Hochspezialisiertes Wissen und Können ist zur Analyse notwendig, aber je länger je weniger hinreichend. So liegt denn auch die größte Forschungslücke in den Regionalwissenschaften in den kausalen Wechselwirkungen zwischen den fünf Feldern in Abb. 1.

Dies dürften vier notwendige Voraussetzungen dafür sein, daß Regionalforschung nicht nur eine Chance für die Geographie darstellt, sondern daß das Fach auch fähig wird, diese Chance zu nutzen. Hoffnungsvolle Ansätze liegen vor; ihrer systematischen Weiterentwicklung sollte große Beachtung geschenkt werden.

\section{Literatur}

BARTELS D. 1977: System, Theorie und Methode der Geographie. Raumwissenschaftliche Aspekte sozialer Disparitäten. In: Mitt. der österr. geogr. Gesellschaft, Bd. 119. S. 227-242.

BRUGGER E.A. (Hrsg.) 1984: Innovationsorientierte Regionalpolitik. Diessenhofen. 
BRUGGER E.A. 1982: Regionale Strukturpolitik in Entwicklungsländern. Diessenhofen.

BRUGGER E.A. 1981: Regionalforschung als Chance der Wirtschafts- und Spezialgeographie. In: Geogr. Helvetica, 4/81, S. 167-175

EISEL U. 1981: Zum Paradigmawechsel in der Geographie. In: Geogr. Helvetica 4/81, S.176-189.

NYDEGGER A./OBERHÄNSLI H./HARRINGER R. 1983: Investitionen und Innovationen in kleineren Industriebetrieben. Diessenhofen.
MAILLAT D. (Ed.) 1983: Technology - a key factor for regional development. St. Saphorin.

RAPOPORT A. 1982: Gegenüberstellung von zwei Ansätzen zur allgemeinen Systemtheorie. Vortragsmanuskript. Wien.

STIENS G. 1980: Zur Wiederkunft des Regionalismus in den Wissenschaften. Informationen zur Raumentwicklung 5/80, S. 315-333.

STUCKEY B. 1980: Economic Progress: Illusion and reality. In: Ethnologie im Dialog, hrsg. von G.BAER/P. CENTLIVRES. Fribourg. S. 135-180.

\subsection{Marktlücken als Chancen für Geographieabsolventen?}

\begin{abstract}
Wenn wir von den Schwierigkeiten der Stellensuche hören, die ausstudierte Geographen haben, wenn sie ins Berufsleben eintreten wollen - um in ihrem Fachgebiet zu arbeiten, wohlgemerkt -, müssen wir uns die Frage stellen, ob ein Bedarf an Geographen überhaupt vorhanden ist. Sicher war die Situation vor ein paar Jahren schlimmer; heute mit steigendem Umweltbewußtsein erhält auch die Stellung des Geographen mehr Bedeutung. Allerdings ist auch er nicht darum herumgekommen, sich zu spezialisieren. Die prekäre Stellensituation hat nun im Geographischen Institut der Universität Bern dazu geführt, da $\beta$ in verschiedenen Abteilungen (v. a. der physikalischen Richtung) Initiativen ergriffen wurden, um in eigenwirtschaftlicher Verantwortung in den speziellen Gebieten selber Arbeitsplätze zu schaffen. METEOTEST darf sich das Verdienst gutschreiben, bei den ersten gewesen zu sein, die solche Pläne konkret umgesetzt haben. Später haben Geomorphologen nachgezogen; es wird eine kleine Firma betrieben, die sich auf Luftbildaufnahmen mit einer $6 \times 6$-Kamera spezialisiert hat; und neuerdings haben auch Hydrologen damit begonnen, auf privater Basis zu arbeiten.

Es scheint also, daß Marktlücken erstens existieren und daß sie zweitens von Geographen als Chancen aufgefaßt werden, in ihrem Beruf tätig zu sein.
\end{abstract}

\subsubsection{Als Beispiel: METEOTEST}

Der Wunschtraum eines jeden Studenten ist es, das an der Universität vermittelte theoretische Wissen auch praktisch anwenden zu können.
So war auch in der Klimaabteilung des geographischen Instituts der Universität Bern (GIUB) schon lange die Idee aufgekommen, ein auf privatwirtschaftlicher Basis geführtes «Büro für angewandte Klimatologie» zu gründen. Man rechnete sich einige Chancen aus, auf dem Gebiet der angewandten Klimatologie einen Marktanteil «ergattern» zu können. Dieser Optimismus beruhte nicht zuletzt auf der Tatsache, da $\beta$ häufig von privater Seite oder von amtlichen Stellen an die Klimatologen des GIUB kleinere oder auch größere bezahlte Aufträge herangetragen wurden. Meist scheiterte deren Ausführung jedoch daran, da $\beta$ sie entweder relativ dringend waren und kurzfristig kein Personal zur Verfügung stand oder daß sie zu weit abseits der allgemeinen Forschungsrichtung standen und deshalb von vornherein abgelehnt wurden.

Zu Beginn des Jahres 1981 begannen sich deshalb einige Angehörige der Klimaabteilung des GIUB daranzumachen, die Gründung eines solchen privaten Büros voranzutreiben. Man war sich einig darüber, eine von der Universität unabhängige Firma aufbauen zu wollen. Sie sollte auch eine rechtliche Form erhalten; wir entschlossen uns, eine Genossenschaft mit Namen METEOTEST zu gründen, die unter diesem Namen ein Büro für angewandte Klimatologie betreiben sollte. In diesem Sinn schufen wir Statuten

Martin Baumgartner, Jürg Engel

Meteotest, Büro für angewandte Klimatologie, Hallerstrasse 50 , 3012 Bern 